\section{Tief einatmen! - leichter gesagt und getan}

\author{
Ein neuartiger Pulver-Inhalator zeichnet sich durch einen geringen \\ Luftwiderstand beim Einatmen aus. Dadurch lassen sich höhere \\ inspiratorische Flussraten und damit auch höhere Depositionsraten \\ von inhalativen Steroiden erzielen.
}

W eil Fluorchlor-Kohlenstoff-Verbindungen als potente Ozonkiller aufgrund gesetzlicher Bestimmungen ersetzt werden sollen, sind PulverInhalatoren als Alternativen für die Steroid-Inhalation von Interesse. Hier gelangt eine definierte Menge Steroidhaltigen Pulvers nicht mittels eines Treibgases in die Lunge, sondern durch einen Luftstrom, den der Patient selbst beim Einatmen erzeugt. Freilich ist damit das Risiko verbunden, dass bei geringer inspiratorischer Flussrate zu wenig Substanz inhaliert wird. Der neue Pulverinhalator $\left(\right.$ Novolizer $\left.^{\circledR}\right)$ ist auf einen möglichst geringen Luftwiderstand beim Einatmen optimiert, so dass
Asthma-Patienten inspiratorische Flussraten von etwa $90 \mathrm{l} / \mathrm{min}$ erzielen können.

In einer Cross-over-Studie testeten 13 gesunde Probanden diesen Inhalator und verglichen ihn mit einem Turbohaler-Pulverinhalator: Die insgesamt vier Versuche pro Proband (mit dem Turbohaler bei einer Flussrate von $58 \mathrm{l} / \mathrm{min}$, mit dem Novolizer bei 54, 65 und 99 $1 / \mathrm{min}$ ) waren jeweils durch eine mindestens 44-stündige Auswasch-Periode getrennt. Wieviel von der 200- $\mu$ g-Dosis des inhalierten, mit 99mTechnetium markiertem Budesonids tatsächlich in der Lunge ankam, wurde szintigraphisch bestimmt:
Mit dem Turbohaler (Flussrate 58 1/min) gelangten 21,4\% der BudesonidDosis in die Lunge; mit dem Novolizer und der vergleichbaren Rate von 54 $1 / \mathrm{min}$ waren es $20 \%$. Inhalierten die Probanden mit dem Novolizer aber mit einer Flussrate von 65 1/min, so brachten sie es im Median auf 25\%, bei der maximalen Flussrate von 99 1/min auf $32,1 \%$. Die regionale Verteilung des Steroids unterschied sich nicht.

\section{Fazit}

Mit dem Novolizer ist die Deposition des inhalierten Wirkstoffs also mindestens so gut wie mit dem Turbohaler. Weil die Konstruktion den Luftwiderstand minimiert, kann der Patient darüber hinaus die Flussrate und dadurch auch die Deposition noch erheblich verbessern.

jfg

Newman SP et al: Scintigraphic comparison of budesonide deposition from two dry powder inhalers. Eur Respir J 2000; 16: $178-83$

\section{Interleukin-4 (IL-4) in der Hauptrolle}

IL-4 spielt eine Schlüsselrolle in der Entzündungspathogenese bei Asthma bronchiale.

In Gegenwart von IL-4 differenzieren naive T-Lymphozyten zu Th2-Zellen, welche wiederum IL-4, IL-5, IL-9 und IL-13 sezernieren und so die Th-2-Antwort verstärken.

IL-4 verstärkt die IgE-vermittelte Immunantwort durch Hochregulation des hochaffinen IgE-Rezeptors (FCeRI) auf Mastzellen und Basophilen und des niedrigaffinen IgE-Rezeptors (FCeRII), u.a. auf B-Lymphozyten und Monocyten.

IL-4 induziert die Expression des "Vascular Cell Adhesion Molecule 1" (VCAM-1), welches die Einwanderung von T-Lymphozyten, Monozyten und Makrophagen sowie Eosinophilen an den Ort des entzündlichen Geschehens bewirkt.

II-4 verlängert durch Hemmung der Apoptose das Überleben der Eosinophilen und bewirkt durch Induktion der Expression des Muzin-Gens eine vermehrte Schleimproduktion in den Bronchien.

\title{
IL-4-Rezeptoren statt inhalativer Steroide?
}

In einer randomisierten, Plazebo-kontrollierten Doppelblind-Studie

konnten amerikanische Forscher zeigen, dass die Inhalation

Iöslichen IL-4-Rezeptors nach Absetzen inhalativer Kortikosteroide vor einer Verschlechterung der Atemwegssymptome schützt.

W issenschaftler aus den USA prüften die Wirkung einer IL-4Blockade durch einen inhalativen, löslichen IL-4-Rezeptor.

Nach Absetzen von inhalativen Steroiden inhalierten 62 Studien-Teilnehmer ein Mal pro Woche 0,75, 1,5 oder 3,0 mg IL-4-Rezeptor oder Plazebo über drei Monate. Während die relative Einsekundenkapazität in der Plazebo-Gruppe um 13\% abfiel, betrug der Rückgang unter 3,0 mg IL-4-Rezeptor nur $2 \%(\mathrm{p}=0,05)$. Auch gingen die täglich von den Patienten gemessenen FEV1-Werten in der Plazebo-Gruppe verglichen mit der mit 3,0 mg behandelten Gruppe signifikant zurück. Die Wirkungen der 0,75- bzw. 1,5-mgDosierungen unterschieden sich nur wenig von Plazebo. Die IL-4-RezeptorTherapie erwies sich als gut verträglich.

\section{Fazit}

Lösliche IL-4-Rezeptoren könnten in Zukunft bei der Behandlung des Asthma bronchiale eine Alternative zur Therapie mit inhalativen Steroiden darstellen. Die Ergebnisse der Studie sind vielversprechend, allerdings sind noch intensive weitere Untersuchungen erforderlich, ehe diese Therapieform in die klinische Praxis eingeführt werden kann. Dr. A. Niedermeier, München

Borish LC et al. Efficacy of soluble IL-4 receptor for the treatment of adults with asthma. J Allergy Clin Immunol 2001; 107: 963-70 